\title{
Differential Effects of In Vitro Treatment with Cinobufotalin on Three Types of Ovarian Cancer Cells
}

\author{
SYEDA H. AFROZE ${ }^{1}$, CHANDER PEDDABOINA ${ }^{1}$, ANTHONY B. MCDOWELL JR. ${ }^{2}$, \\ A.H.M. ZUBERI ASHRAF ${ }^{2}$, TIMOTHY C. MCCORMICK ${ }^{2}$, M. KAREN NEWELL-ROGERS ${ }^{1}$, \\ DAVID C. ZAWIEJA ${ }^{1}$, THOMAS J. KUEHL ${ }^{2}$ and MOHAMMAD N. UDDIN ${ }^{1,3}$ \\ ${ }^{1}$ Department of Medical Physiology, Texas A\&M University College of Medicine, Temple, TX, U.S.A; \\ ${ }^{2}$ Baylor Scott \& White Health, Temple, TX, U.S.A.; \\ ${ }^{3}$ Orion Institute for Translational Medicine, Temple, TX, U.S.A.
}

\begin{abstract}
Background/Aim: Cinobufotalin (CINO), a cardiotonic steroid, has been used as an anticancer agent. This study assessed the cell-specific effect of CINO on SK-OV-3, CRL-1978 and CRL-11731 ovarian cancer cells which differ in terms of their respective karyotypes. Materials and Methods: Cell cultures were treated with CINO $(0.1,1$, 5 and $10 \mu \mathrm{M})$ for 24,48 , and $72 \mathrm{~h}$. Cell proliferation, migration, and invasion were measured using CellTiter, Cytoselect, and FluoroBlock assays, respectively. Expression of proliferating cell nuclear antigen (PCNA) was evaluated by western blot analysis. Cell viability was determined by fluorescence-activated cell sorting. Immunofluorescence was performed using Annexin-V staining and fluorescein isothiocyanate (FITC). Mitochondrial membrane potential (MMP) was measured using MitoTracker ${ }^{T M}$ Red. Results: CINO at $0.5 \mu M$ inhibited SK-OV-3, CRL-1978, and CRL11731 proliferation, migration, and invasion. Each cell type differed in response to CINO doses for PCNA, Annexin-V expression and MMP. Conclusion: The antineoplastic property of CINO is consistent, but its mode of action varies among cell lines.
\end{abstract}

Ovarian cancer is a relatively rare type of cancer, accounting for only $1.3 \%$ of all new cancer cases per year in the United States $(1,2)$. Despite this percentage, it is deadly, ranking as the sixth leading cause of all cancer-related deaths and the

Correspondence to: Mohammad N. Uddin, Ph.D., FAHA, Executive \& Scientific Director, Orion Institute for Translational Medicine, Temple Health \& Bioscience District, Room \# 109, 1802 S. 1st Street, Temple, TX 76504, U.S.A. Tel: +1 2545439488, e-mail: nasir.uddin@oriontranslational.org

Key Words: Ovarian cancer cell lines, cinobufotalin, bufadienolide, migration, viability, apoptotic signaling, mitochondrial membrane potential. number one cause of gynecological cancer deaths (3). The 5 -year survival rate only improved from $33.7 \%$ to $44.1 \%$ from 1975 to 2012, while that for patients with breast cancer improved from $75.2 \%$ to $91.0 \%$ during the same interval (1, 2 ). Development of better screening tools and discovery of cell-specific treatments are key to changing these statistics.

Little progress has been made in early detection and diagnosis of ovarian cancer. Most women do not experience pain or symptoms until an advanced stage of the disease. Symptoms are often vague, including pelvic discomfort, bloating, and varying urinary or bowel patterns (4). One study attempted screening women with six annual cancer antigens 125 (CA-125) assays and four annual transvaginal ultrasound examinations but did not demonstrate a reduction in ovarian cancer deaths (4). Current recommendations advise that only women with confirmed mutations of breast cancer resistant genes (BRCAl) or $B R C A 2$ who have not undergone prophylactic bilateral salpingo-oophorectomy should undergo such screening tests (4-6). The complexity of ovarian tissue has resulted in numerous subtypes of cancer that fall under the general term 'ovarian cancer'. Epithelial ovarian cancer is by far the most common type of ovarian cancer, representing over $90 \%$ of cases (7). This category is further subdivided into serous, mucinous, endometrioid, clear cell, and transitional cell tumors $(8,9)$. The diversity of these tumors and their cell origins make the discovery of novel and biologicallyspecific treatments extremely difficult, yet important for improvement of survival of patients with ovarian cancer. Current treatment methods consist of debulking surgery and platinum-based chemotherapy (carboplatin and paclitaxel) for epithelial cell tumors and combination bleomycin, etoposide and cisplatin chemotherapy for germ cell tumors $(8,10-12)$. Development of novel therapies that act in a cell-specific manner could significantly alter prognosis and improve post-treatment outcomes of those diagnosed with ovarian cancer. 
Recent research has focused on utilizing compounds isolated from plants and animals for medicinal purposes, especially those that have been used in traditional Chinese medicine. Cinobufotalin is a bufadienolide with diuretic and hemostatic properties and has been used as a cardiotonic steroid $(3,13,14)$. Remarkably, further research has revealed a cytotoxic effect of cinobufotalin that may be beneficial in treating several cancer types such as lymphoma, lung cancer, and ovarian cancer in a cell-specific manner $(15,16)$. Cinobufotalin was found to induce apoptosis in U937 human lymphoma cells through DNA fragmentation, decrease of mitochondrial membrane potentiaI (MMP), increase in intracellular $\mathrm{Ca}^{2+}$, and increase in production of reactive oxygen species, ultimately leading to activation of apoptotic proteins $(8,16-18)$. Our research is focused on applying the known cytotoxic features of cinobufotalin to three different ovarian cancer cell lines (SK-OV-3, CRL-1978, CRL-11731). SK-OV-3 is a hypodiploid human cell line with a modal chromosome number of 43 (occurring in $63.3 \%$ of cells) and a range of 42 to 45 . The rate of higher ploidies is $32 \%$. One $\mathrm{N} 11$ has the homogeneously staining region (HSR) segment from p11 to the distal end. The normal N10, N12, N15, N17 and N19 are absent. CRL-1978 cell line has a complex hyper diploid karyotype of $66 \mathrm{XX}$ to $88 \mathrm{XX}$. These cells exhibit low to moderate resistance to several chemotherapeutic agents including doxorubicin, cisplatin, carmustine, etoposide and cyanomorpholinodoxorubicin (MRA-CN) and also express low levels of P glycoprotein. The karyotype of CRL-11731 cell line is still not well known. Morphologically it is an epithelial cell line from a French-Canadian ancestry patient with early onset ovarian cancer. A recent study from our laboratory demonstrated that cinobufotalin at in vitro concentrations greater than $0.5 \mu \mathrm{M}$ inhibited SK-OV-3 cell proliferation, migration and invasion (19). The goal of this study was to determine if the cytotoxic effect of cinobufotalin is cell-specific or if it affects each cancer cell line similarly. Additionally, we also aimed to compare the mechanism of action of cinobufotalin on each of the cell lines.

\section{Materials and Methods}

Cell culture. Three ovarian cancer cell lines, CRL-1978, CRL-11731 and SK-OV-3 (American Type Culture Collection, Manassas, VA, USA), were grown in ATCC-formulated McCoy's 5a Medium (Thermo Fisher Scientific, Waltham, MA, USA) supplemented with $10 \%$ Gibco fetal bovine serum (FBS) (Thermo Fisher Scientific) and penicillin-streptomycin-amphotericin B cocktail (Thermo Fisher Scientific). Cells were cultured at $37^{\circ} \mathrm{C}$ in a humidified atmosphere of $5 \% \mathrm{CO}_{2}$. Cinobufotalin, was purchased from Enzo Life Sciences (Farmingdale, NY, USA) and diluted in dimethyl sulfoxide (DMSO) for in vitro treatments. DMSO at $0.2 \%$ concentration was also used as control.

Cell proliferation assay. Cell proliferation was measured using a CellTiter Assay (Promega, Madison, WI, USA). Cells were treated with $0.1,0.5,1,5$, and $10 \mu \mathrm{M}$ cinobufotalin for $72 \mathrm{~h}$. Afterwards, CellTiter 96 was added to the cells, and absorbance at $490 \mathrm{~nm}$ was measured using a plate reader (SPECTRAmax 340PC384; Microplate Spectrophotometer, Molecular Devices LLC, Sunnyvale, CA, USA). The absorbances were graphed against concentration of cinobufotalin treatments to determine the effect of the compound on cancer cell proliferation.

Cell migration assay. Cell migration was measured using a CytoSelect Assay (Cell Biolabs, San Diego, CA, USA). Cells were treated with $0.1,0.5,1,5$, and $10 \mu \mathrm{M}$ cinobufotalin and incubated for 24 hours at $37^{\circ} \mathrm{C}$ in $5 \% \mathrm{CO}_{2}$. Cells in serum-free medium were dispensed inside the insert, while serum medium was placed into the well. Migratory cells travelled through the pores in the direction of the serum medium in the well and could be stained or measured on a plate reader. CyQuant GR Dye solution was then added to the cells and fluorescence measured at $480 \mathrm{~nm} / 520 \mathrm{~nm}$ on a fluorescence plate reader (CytoFluor Series 4000 Fluorescence Multi-Well Plate Reader; Applied Biosystems, Foster City, CA, USA). The migratory cells were quantified by measuring their absorbance and plotted against treatment concentrations to determine the effect of cinobufotalin on cell migration.

Cell invasion assay. Cell invasion was measured using a FluoroBlok Assay (BD, Franklin Lakes, NJ, USA). Cells were treated with 0.1, $0.5,1,5$, and $10 \mu \mathrm{M}$ cinobufotalin for 48 hours at $37^{\circ} \mathrm{C}$ in $5 \% \mathrm{CO}_{2}$. This assay included a FluoroBlok 24-Multiwell Insert Plate with 8.0-micron pore size PET membrane coated with BD Matrigel Matrix. This coating method obstructed the membrane's pores, hindering the migration of non-invasive cells from passing through the membrane. Calcein-acetoxymethyl was added to the cells and fluorescence was measured at $490 \mathrm{~nm} / 700 \mathrm{~nm}$ on a fluorescence plate reader (CytoFluor Series 4000; Applied Biosystems) to quantify invasive cells and plot them against treatment concentrations.

Cell viability assay. Cell viability was measured using a CellTiter Assay (Promega, Madison, WI, USA). This assay measured conserved and constitutive protease activity inside living cells. The live-cell protease activity is limited to complete viable cells and was measured using a fluorogenic, cell-permeant, peptide substrate. The substrate enters undamaged cells, where it is divided by the livecell protease activity to generate a fluorescent signal comparative to the quantity of living cells. The live-cell protease is inactivated upon the loss of membrane stability and outflow into the surrounding culture medium. Cells were treated with $0.1,0.5,1,5$, and $10 \mu \mathrm{M}$ cinobufotalin for $48 \mathrm{~h}$ at $37^{\circ} \mathrm{C}$ in $5 \% \mathrm{CO}_{2}$. CellTiterBlue reagent was then added to the cells and absorbance was measured at $520 \mathrm{~nm}$ on a plate reader (SPECTRAmax 340PC384; Molecular Devices LLC) and plotted against treatment concentrations.

Cell lysis and total protein estimation. Cells were treated with 0.1 , $0.5,1,5$, and $10 \mu \mathrm{M}$ cinobufotalin for 48 hours at $37^{\circ} \mathrm{C}$ in $5 \% \mathrm{CO}_{2}$. Cells were then lysed using lysis buffer (Cell Signaling Technology, Danvers, MA, USA). Cells were then scraped into tubes and spun down to collect the supernatant. Protein samples were then loaded onto a 96-well plate, and protein estimation was determined using standards from a bicinchoninic acid reagent kit (Pierce, Rockford, IL, USA). The standards were used to produce a standard curve 
from which the concentration of cellular protein from cinobufotalintreated cells was determined.

Western blot assay to determine proliferating cell nuclear antigen (PCNA) expression. Using the protein estimation data, each sample was prepared at a protein concentration of $10 \mu \mathrm{g} / \mu \mathrm{l}$, including deionized $\mathrm{H}_{2} \mathrm{O}$, NuPage 4x LSD Sample buffer and NuPage 10x Reducing Agent (Invitrogen, Carlsbad, CA, USA). Prepared samples were loaded into a NuPAGE Novex 4-12\% Bis-Tris gel (Invitrogen) and transferred onto a nitro-cellulose membrane (Invitrogen). Membranes were blocked in 5\% milk and on separate occasions probed with antibodies to PCNA and $\beta$-actin (Santa Cruz, Dallas, TX, USA). After incubation with the corresponding secondary antibody, proteins were visualized with chemiluminescence detection kit (Pierce, Thermo Fisher Scientific, Carlsbad, CA, USA). The intensity of the bands was determined by imaging the membranes in an ImageQuant LAS 4000 instrument (GE Healthcare, Life Sciences, Pittsburgh, PA, USA). The expression of PCNA protein was quantified by densitometric analysis using Image J (NIH, Bethesda, MD, USA).

Fluorescence Activated Cell Sorting (FACS) analysis for cell viability and apoptosis. Cell viability was additionally evaluated by a Cell Viability Kit (Cayman Chemical, Ann Arbor, MI, USA) and apoptosis was evaluated using an Apoptotic Blebs Assay Kit (Cayman Chemical). Cells were treated with $0.1,0.5,1,5$, and $10 \mu \mathrm{M}$ cinobufotalin for $48 \mathrm{~h}$. The cell viability and apoptotic blebs assay was performed on a BD FACSCanto II FACS analyzer (BD Biosciences, San Jose, CA, USA) and the data analysis was performed using FlowJo (Ashland, OR, USA). Results were expressed as a percentage of live and dead cells.

Annexin-V staining. SK-OV-3, CRL-1978, and CRL-11731 cells were seeded on coverslips in 6-well plates and allowed to adhere overnight. The cells were then treated with $0,0.5$, and $5 \mu \mathrm{M}$ cinobufotalin for $48 \mathrm{~h}$. Afterwards a mixture of biotinylated Annexin-V (Thermo Fisher Scientific), Cy3-labeled streptavidin binding buffer (GE Healthcare, Life Sciences), and propidium iodide (Thermo Fisher Scientific) was added to the cells on the coverslips. The prepared coverslips were placed on a slide and the nuclear marker 4',6'-diamidino-2-phenylindole (Invitrogen) was added. The slides were observed on an Olympus FluoView FV 300 confocal laser-scanning microscope (Shinjuku, Tokyo, Japan) using the FITC filter to determine expression of stained PS to assess apoptotic signal in the cells.

MMP assay. SK-OV-3, CRL-1978, and CRL-11731 cells were treated in serum-free medium with $0,0.1,0.5,1,5$, and $10 \mu \mathrm{M}$ cinobufotalin for 12 and $24 \mathrm{~h}$. The cells were then stained with MitoTracker $^{\mathrm{TM}}$ Red (M7513; Thermo Fisher Scientific) following the manufacturer's protocol. The stained cells were analyzed on BD FACSCanto II FACS analyzer (BD Biosciences) and FlowJo was used for data analysis to calculate the mean fluorescent intensity (MFI) as a representation of the MMP.

Statistical methods. Data from in vitro experiments of cinobufotalintreated cells were compared to those from DMSO-treated controls using analysis of variance (ANOVA). Experiments were repeated five times. Duncan's post-hoc testing was used to detect differences between treatment groups. Differences with a $p$-value of less than 0.05 were considered significant.

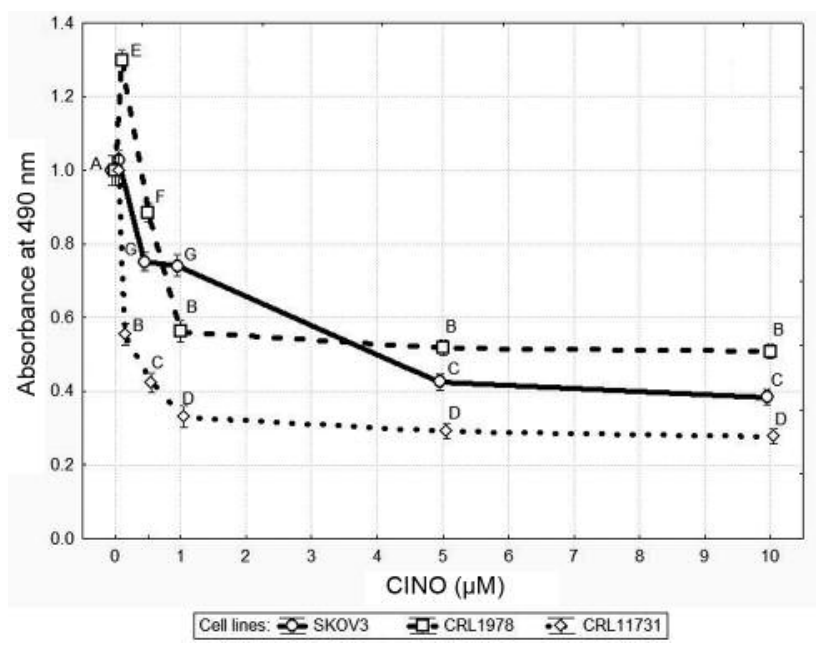

Figure 1. In vitro cell proliferation assay results for $S K-O V-3$, CRL-1978, and CRL-11731 cells evaluated at 48 hours in response to cinobufotalin (CINO) measured as absorbance at $490 \mathrm{~nm}$ normalized to that of the control. Data expressed as means \pm SE for 8 replicates. Cell lines differ ( $p<0.001$ using ANOVA) and means with different letters differ ( $p<0.05$ using the Duncan's post-hoc test).

\section{Results}

Cinobufotalin at in vitro concentrations greater than $0.5 \mu \mathrm{M}$ significantly inhibited cell proliferation, migration, and invasion $(p<0.05)$ of SK-OV-3, CRL-1978, and CRL-11731 ovarian cancer cell lines (Figures 1-3). There appeared to be a dosedependent decrease in proliferation of SKOV-3, CRL-1978, and CRL-11731 in cultures treated with cinobufotalin. There was no significant difference in effect between 5 and $10 \mu \mathrm{M}$ treatment of SK-OV-3 CRL-1978, and CRL-11731 cells.

Figures 2 and 3 show a similar trend of statistically significant decrease in migration and invasion, respectively, at a dose of $0.5 \mu \mathrm{M}(p<0.05)$.

The Western blots assays revealed dose-dependent decrease in expression of PCNA normalized to that of $\beta$-actin in SK-OV3, CRL-1978, and CRL-11731 cells (Figure 4A). The densitometric analysis of the Western blots showed the most pronounced inhibition to occur between 0.5 and $5 \mu \mathrm{M}$ of cinobufotalin for all three cell lines (Figure 4B).

FACS data showed a similar dose-dependent trend of reduced viability at $0.5 \mu \mathrm{M}$ cinobufotalin for CRL1978, but not for SK-OV3 and CRL-11731 cell lines. The viability of the CRL-1978 cells appeared to increase at cinobufotalin concentrations of $0.5 \mu \mathrm{M}$ and more (Figure 5).

Cinobufotalin at $\geq 0.5 \mu \mathrm{M}$ induced apoptotic signaling and led to disintegration of SK-OV-3 (Figure 6A) and CRL-1978 (Figure 6B) cells. CRL-11731 cells did not exhibit the same effects and appeared to be more resistant to the apoptotic effects of cinobufotalin (Figure 6C). 


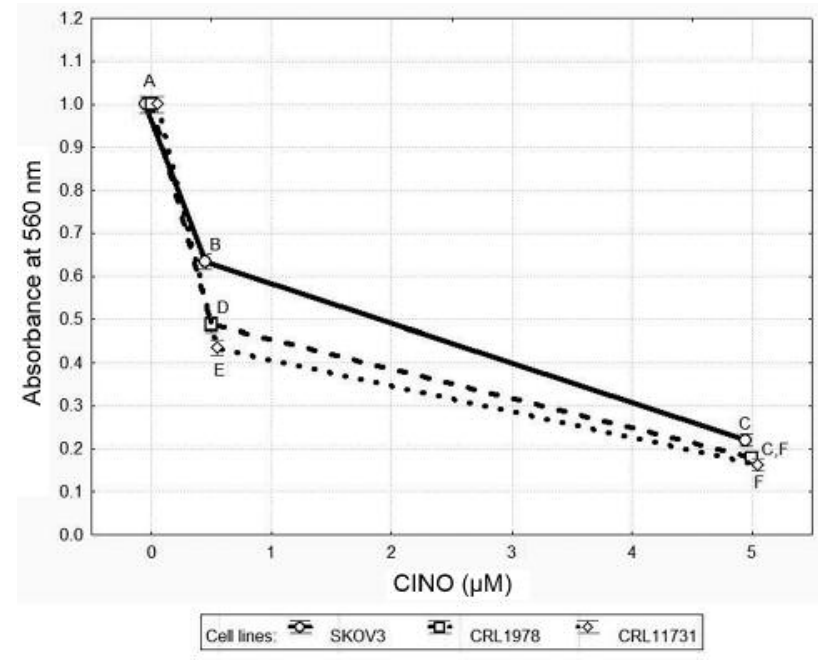

Figure 2. In vitro cell migration assay results for SK-OV-3, CRL-1978, and CRL-11731 cells evaluated at $24 \mathrm{~h}$ migration in response to cinobufotalin (CINO) measured as absorbance at $560 \mathrm{~nm}$ normalized to that of the control. Data expressed as means $\pm S E$ for 8 replicates. Cell lines differ ( $p<0.001$ using ANOVA) and means with different letters differ ( $p<0.05$ using Duncan's post-hoc test).

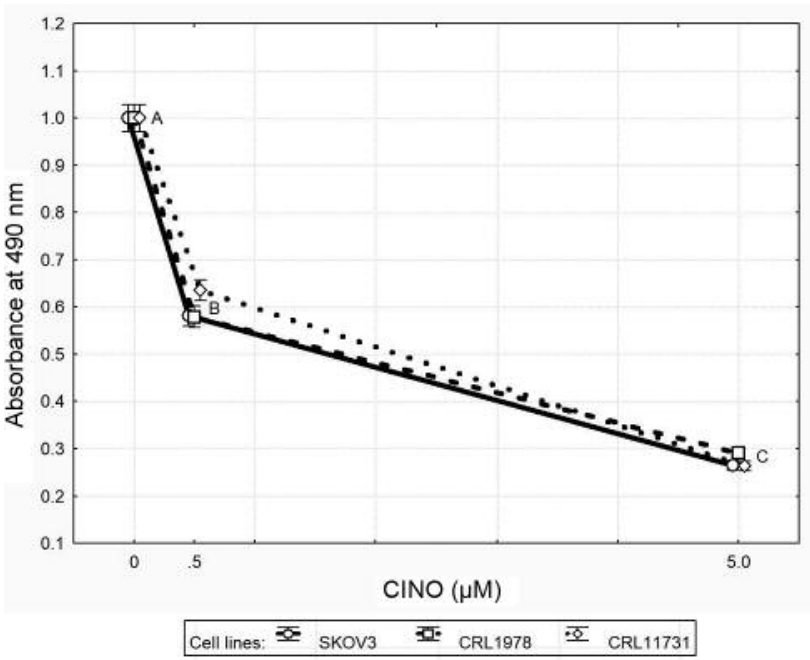

Figure 3. In vitro cell invasion assay results for SK-OV-3, CRL-1978, and CRL-11731 cells evaluated at 48 hours line invasion in response to cinobufotalin (CINO) measured as absorbance at $490 \mathrm{~nm}$ normalized to that of the control. Data expression as means \pm SE for 8 replicates. Cell lines do not differ ( $p=0.58$ using ANOVA), but responses to CINO dosages differ $(p<0.001$ using ANOVA). Means with different letters differ ( $p<0.05$ using Duncan's post-hoc test).

Cinobufotalin at $\geq 1 \mu \mathrm{M}$ reduced the mitochondrial membrane potential (MMP) of SK-OV-3 cells (Figure 7A). On the contrary, the MMP for CRL-1978 and CRL-11731 increased with cinobufotalin treatment (Figure 7B and C, respectively).
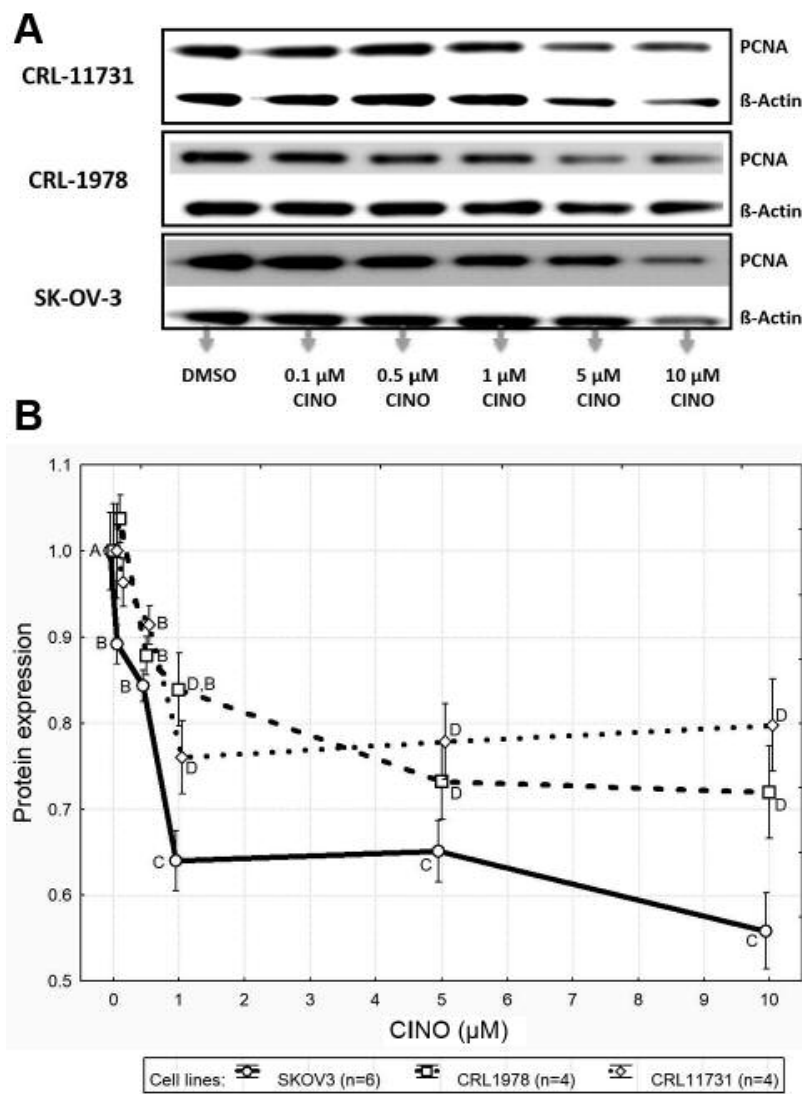

Figure 4. Expression of proliferating cell nuclear antigen (PCNA) expression in SK-OV-3, CRL-1978, and CRL-11731 ovarian cancer cells treated with different concentrations of cinobufotalin (CINO). A: Western blot band images. B: Graphical representation of PCNA expression quantified relative to that of $\beta$-actin normalized to that of the control. Results for SK-OV-3 (n=6), CRL-1978 (n=4), and CRL$11731(n=4)$ cells shown as means \pm SE. Cell lines differ in response to CINO doses ( $p=0.0018$ using ANOVA) and measures for different CINO doses differ $(p<0.0001$ using ANOVA). Means with different letters differ ( $p<0.05$ using the Duncan's post-hoc test.

\section{Discussion}

According to our study, reduced expression of PCNA was observed across all three cell lines on treatment with cinobufotalin. In a prior study by McDowell et al. with SK$\mathrm{OV}-3$ ovarian cancer cells, it was observed that cinobufotalin concentrations exceeding $0.5 \mu \mathrm{M}$ reduced PCNA expression and cell viability and induced apoptotic signaling (19). CRL1978 and CRL-11731 lines exhibited similar trends. PCNA also serves as a co-factor for the several polymerases involved in DNA repair and elongation and its down-regulation diminishes the ability of rapidly dividing cancer cells to repair their DNA $(29,30)$. Cinobufotalin at concentrations $>0.5 \mu \mathrm{M}$ 


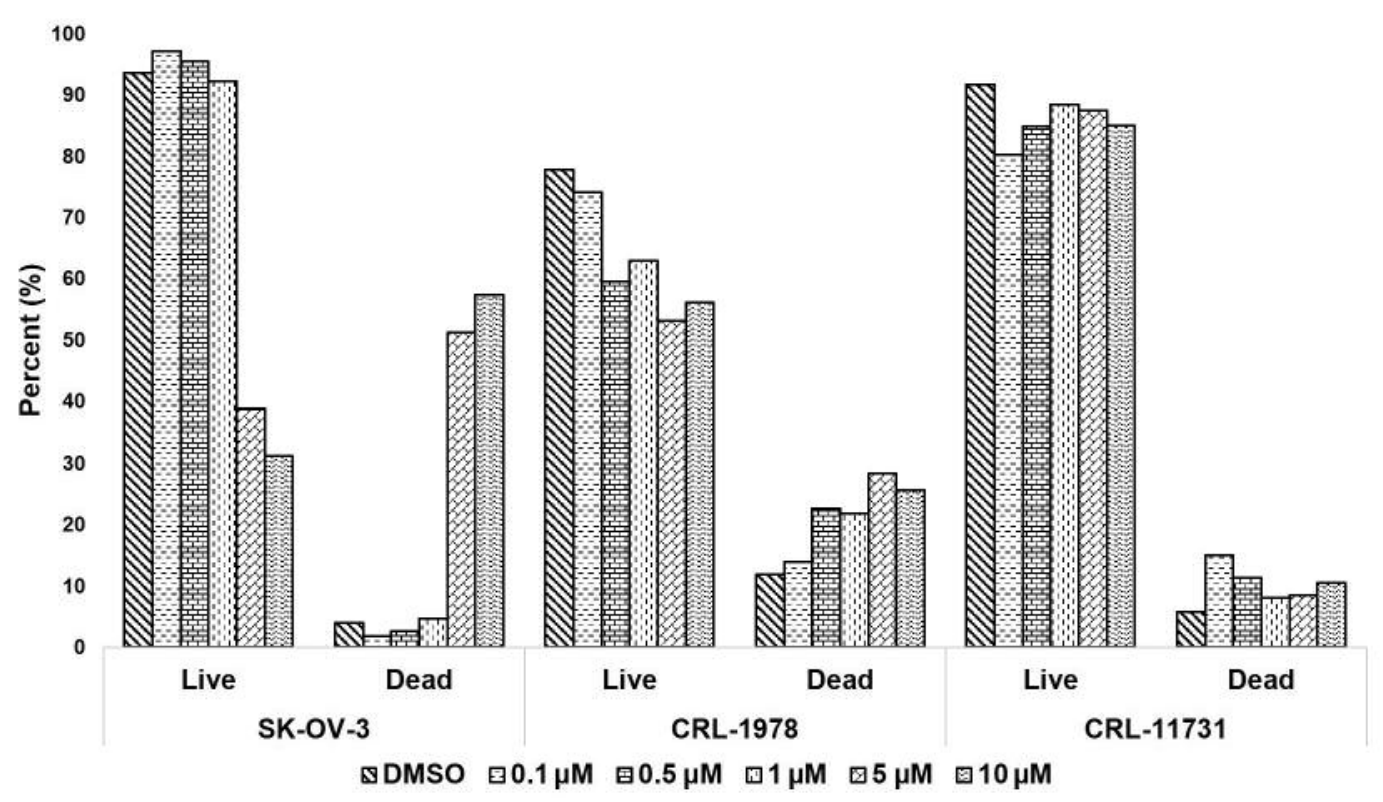

Figure 5. Fluorescent-activated cell sorting (FACS) analysis of live and dead SK-OV-3, CRL-1978, and CRL-11731 cells in response to different concentrations of cinobufotalin (CINO).

inhibited proliferation, migration, and invasion of all three different ovarian cancer cell lines, in vitro (SK-OV-3, CRL1978, and CRL-11731). Annexin-V is used as a qualitative probe to detect cells that have phosphatidylserine on the cell surface, an event found during apoptosis as well as other forms of cell death. Treatment of both SK-OV3 and CRL1978 cell lines with cinobufotalin concentrations $>5 \mu \mathrm{M}$ led to increased staining of annexin- $\mathrm{V}$ which correlates with increased apoptotic signaling. On the other hand, CRL-11731 seemed to be less affected in terms of apoptosis. This cell line is known to have p53 mutation, which would be consistent with its decreased entry into apoptosis. Cinobufotalin was shown to induce both the intrinsic and extrinsic apoptotic pathways through the increased expressions of bcl-2 associated $\mathrm{X}(\mathrm{BAX})$ protein, $\mathrm{BAX}$ like $\mathrm{BH} 3$ protein, cytochrome $c$, caspase-2, $-3,-8,-9$ and FAS receptor in a human lymphoma cell line (U937) (15). To further investigate the nature of apoptotic signaling, we evaluated the MMP in all three cell lines. SK-OV-3 was shown to have loss of MMP with increased treatment concentration. This finding is consistent with the intrinsic pathway and formation of pores in the mitochondrial membrane. The results, however, contrasted with the CRL-11731 and CRL-1978 cell lines, which displayed the opposite, where the MMP for these cell lines increased with increasing concentration of cinobufotalin.

Several studies have been carried out to address the mechanism of action for this compound's anti-tumor effects. DNA fragmentation decreased MMP, apoptotic protein activation, up-regulation of FAS protein, and activation of cytochrome $c$ has been proposed (5). Recently we showed that cinobufotalin impaired cytotrophoblast cell function via cellcycle arrest (24). Zhao et al. isolated cinobufotalin from Bufo gargarizans (Asiatic toad) and showed it to have significant inhibitory effects against a human hepatocarcinomatous cell line (smmc7721) in vitro (28). The trend of differing mechanism of actions of cinobufotalin appears to be consistent, even in ovarian cancer cells. This is likely secondary to the heterogeneous distribution of $\mathrm{Na}^{+} / \mathrm{K}^{+}$ATPase throughout the body, as well as the complexity of the downstream pathways from this enzyme and the pro-apoptotic signaling cascades that cinobufotalin modulates (30). Binding to and inhibition of $\mathrm{Na}^{+} / \mathrm{K}^{+}$ATPase can lead to increased cytosolic sodium and decreased potassium levels, which in turn increases cytosolic calcium and as a result causes mitochondrial $\mathrm{Ca}^{2+}$ overload, respiratory inhibition, and mitochondrial dysfunction due to changes in mitochondrial permeability (31). Furthermore, cathepsin S binding to this enzyme is known to directly activate a signaling cascade involving mitogen-activated protein kinase, phosphoinositide 3-kinase, protein kinase $\mathrm{B}$, and protein kinase $\mathrm{C}$, all of which lead to changes in gene expression (30). Emam et al. tested the effects of cinobufotalin on human lymphoma U937 cells and HeLa cells and found that cinobufotalin induced caspasedependent apoptosis (15). They investigated the intrinsic and extrinsic pathway of apoptotic signaling in response to cinobufotalin. In the intrinsic pathway, cinobufotalin caused a decline in MMP while releasing cytochrome $\mathrm{c}$ and activating caspase-3 leading to DNA fragmentation and cell death. In the 
A

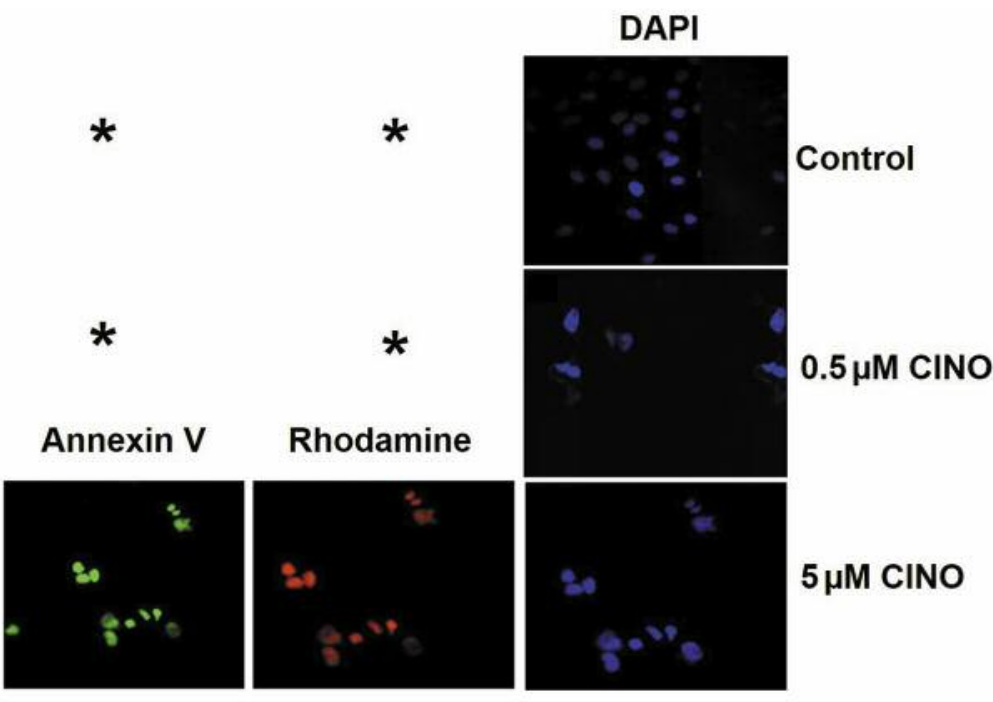

B
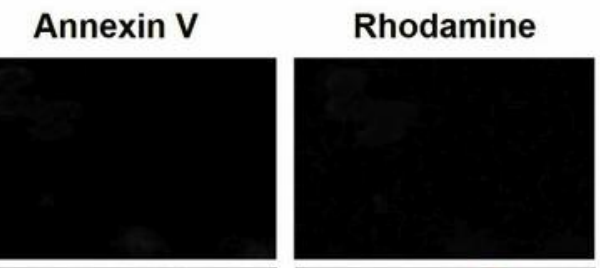

DAPI
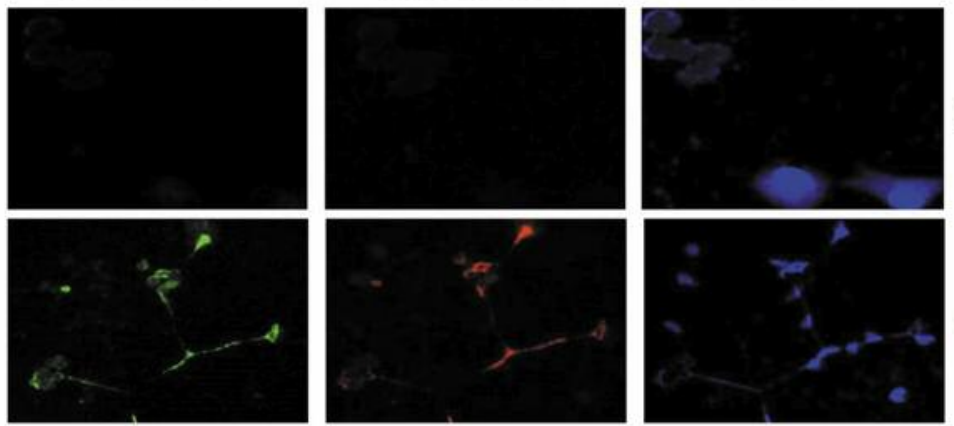

Control
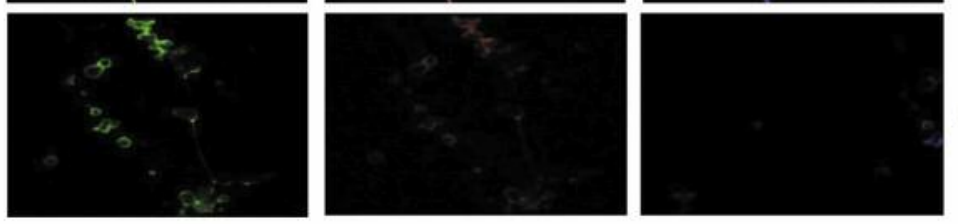

$0.5 \mu \mathrm{M}$ CINO

C

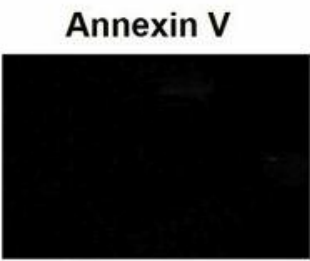

Rhodamine

DAPI
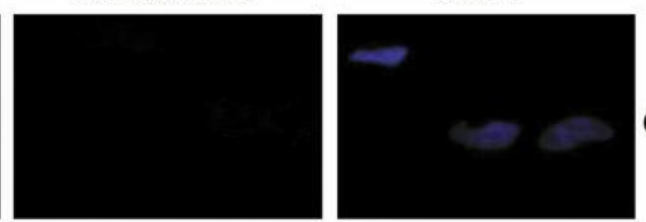

Control
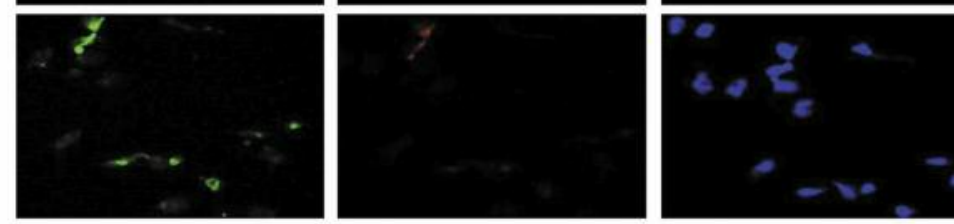

$0.5 \mu \mathrm{M}$ CINO
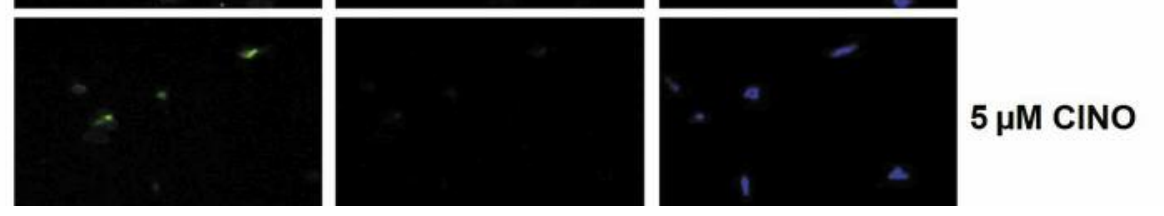

Figure 6. Determination of apoptotic signaling in A: SK-OV-3, B: CRL-1978 and C: CRL-11731 cell lines in response to different concentrations of cinobufotalin (CINO) by Annexin V and DAPI staining. Rhodamine is used as a control stain. *Images not available due to scheduling conflicts of microscopy facilities. 


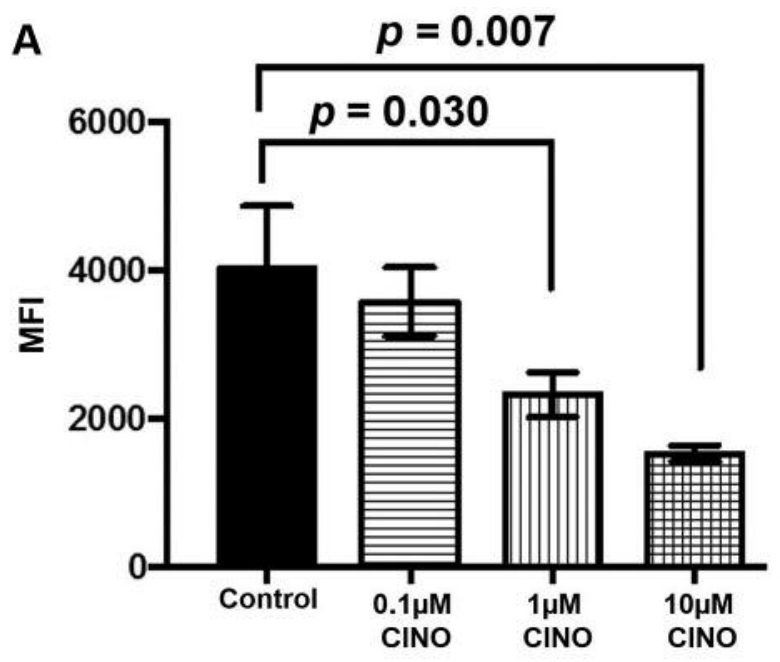

B
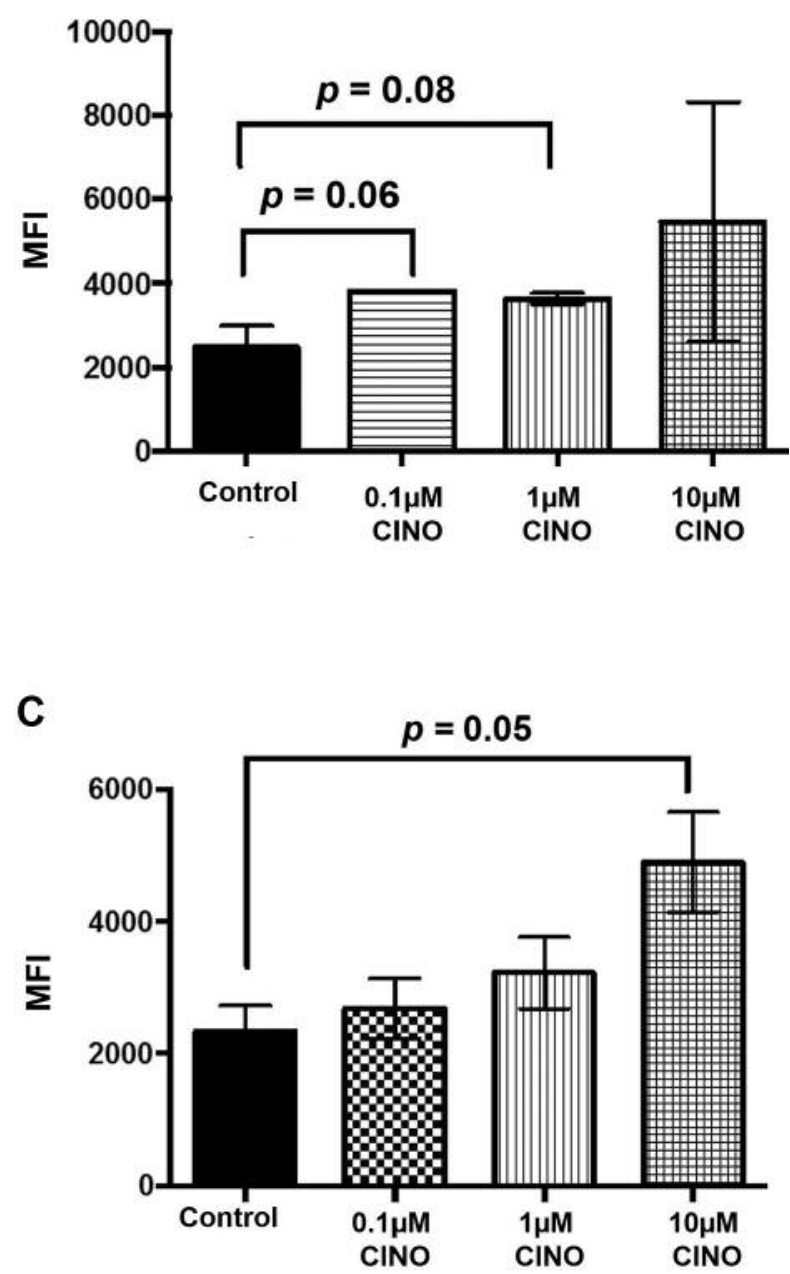

Figure 7. Determination of mitochondrial membrane potential in A: SK-OV-3, B: CRL-1978 and C: CRL-11731 cell lines in response to cinobufotalin (CINO) by measuring the mean fluorescent intensity (MFI) against different treatment concentrations of CINO. extrinsic pathway, cinobufotalin has caused the upregulation of a transmembrane protein called FAS initiating an intracellular signaling cascade leading to the formation of death inducing signaling complex (DISC). DISC activates procaspase- 8 leading to the activation of caspase- 3 followed by DNA fragmentation and cell death (15). These mechanisms demonstrate the wide variety of biochemical pathways affected by cinobufotalin. Thus, the complexities, along with the karyotypic differences between varying cancer cell lines, supports the diversity of mechanisms that were proposed earlier. Studies evaluating the effect of cinobufotalin on primary cancer cell isolates, and in vivo effects on tumor growth in mice are now underway in our laboratory.

Indran et al. mentioned in their findings that defective or inefficient apoptosis is a known attribute of cancer cells (23). Cancer tends to have a hyperproliferative nature and emerging complexities in terms of molecular and functional mechanisms which has limited the ways to effectively treat cancer (10). Exploring the nature and mechanism of action will be very useful when testing a potential drug to be considered for cancer treatment. In addition, a thorough understanding of apoptotic signaling, its pathways, and possible mechanism of apoptotic resistance, and how our potential drug influence apoptosis is imperative to unravel a novel treatment procedure (23). Recently, compounds extracted from plants, herbs, and other animals have become the forefront of medicinal research to assess their natural benefit against diseases like cancer $(11,13$, 22). When experimentally tested, these compounds displayed anti-oxidative, anti-inflammatory, anti-proliferative, and apoptosis-inducing properties $(18,20-22)$. Thus, we propose that previous studies and our recent findings have shown that cinobufotalin affects different cancer cell signaling and can be a potential therapeutic drug against ovarian cancer $(10,19)$.

\section{Acknowledgements}

Funding for this work was provided by The Discovery Foundation, (MNU) and Grimes Family Foundation Endowment in Gynecologic Oncology (MNU) and Noble Centennial Endowment for Research in Obstetrics and Gynecology (TJK), Baylor Scott \& White Healthcare, Temple, Texas.

\section{Conflicts of Interest}

None of the Authors have any potential conflict of interest in relation to this work.

\section{References}

1 NIH. National Cancer Institute. Surveillance, Epidemiology, and End Results Program. Cancer Stat Facts: Ovarian Cancer. https://seer.cancer.gov/statfacts/html/ovary.html

2 NIH. National Cancer Institute. Surveillance, Epidemiology, and End Results Program. Cancer Stat Facts: Female Breast Cancer. https://seer.cancer.gov/statfacts/html/breast.html 
3 Jayson GC, Kohn EC, Kitchener HC and Ledermann JA: Ovarian Cancer. Lancet 384(9951): 1376-1388, 2014.

4 Berek JS and Hacker NF (eds): Practical Gynecologic Oncology. Lippincott Williams \& Wilkins, 2005.

5 Lambert C, Apel K, Biesalski HK and Frank J: 2methoxyestradiol induces caspase-independent, mitochondriacentered apoptosis in DS-sarcoma cells. Int J Cancer 108(4): 493-501, 2004.

6 Minion LE, Dolinsky JS, Chase DM, Dunlop CL, Chao EC and Monk BJ: Hereditary predisposition to ovarian cancer, looking beyond BRCA1/BRCA2. Gynecol Oncol 137(1): 86-92, 2015.

7 Hennessy GT, Suh GK and Markman M: Chapter 28. Ovarian Cancer. The MD Anderson Manual of Medical Oncology, 2nd ed, McGraw-Hill Global Education Holdings, 2011.

8 Seiden MV: Gynecologic Malignancies. Harrison's Principles of Internal Medicine, 19th ed, McGraw-Hill Global Education Holdings, 2015.

9 Niederhuber JE and Armitage JO: Abeloff's Clinical Oncology Review, 5th ed, Saunders, 2013.

10 Kai S, Lu JH, Hui PP and Zhao H: Pre-clinical evaluation of cinobufotalin as a potential anti-lung cancer agent. Biochem Biophys Res Commun 452(3): 768-774, 2014.

11 Tao C and Dan L: In vivo and in vitro effects of QHF combined with chemotherapy on hepatocellular carcinoma. J Biomed Res 24(2): 161-168, 2010.

12 Hoffman BL, Schorge JO, Bradshaw KD, Halvorson LM, Schaffer JI and Corton MM: Williams gynecology. McGraw Hill Professional, 2016.

13 Feng K, Zhou HJ and Hu YL: Effect of cinobufotalin on growth of xenograft of endometrial carcinoma cell line Ishikawa in nude mouse and its impact on RRM2 expression. Chin J Integr Med 30(11): 1183-1185, 2010.

$14 \mathrm{Xu} \mathrm{K}$, Luo HY and Li LN: Clinical study on comprehensive treatment of primary liver cancer mainly with Chinese medicinal perfusion/embolization. Chin J Integr Med 25(4): 299-302, 2005.

15 Emam H, Zhao QL, Furusawa Y, Refaat A, Ahmed K, Kadowaki $\mathrm{M}$ and Kondo T: Apoptotic cell death by the novel natural compound, cinobufotalin. Chem Biol Interact 199(3): 154-160, 2012.

16 Xin XL, Liu J, Ma XC, Wei Q, Lv L, Wang CY, Yao JH and Cui $\mathrm{J}$ : Preparative separation of four major bufadienolides from the Chinese traditional medicine, Chansu, using high-speed countercurrent chromatography. Natural Prod Commun 5(7): 10311034, 2010.

17 Nasu K, Nishida M, Ueda T, Takai N, Bing S, Narahara H and Miyakawa I: Bufalin induces apoptosis and the $G_{0} / G_{1}$ cell cycle arrest of endometriotic stromal cells: a promising agent for the treatment of endometriosis. Mol Hum Reprod 11(11): 817-823, 2005.

$18 \mathrm{Xu} \mathrm{XT:} \mathrm{Clinical} \mathrm{study} \mathrm{on} \mathrm{cinobufotalin} \mathrm{in} \mathrm{treating} \mathrm{chronic}$ hepatitis B virus carriers. Chin J Integr Med 13(8): 473-475, 1993.

19 McDowell A, Afroze SH, Tobin R, McCormick TC, NewellRogers MK, Zawieja DC, Kuehl TJ and Uddin MN: Cinobufotalin as a novel agent to inhibit in vitro epithelial ovarian cancer cell proliferation, migration and invasion. OJOG 6(06): 343-351, 2016.
20 Uddin MN, Horvat D, Glaser SS, Danchuk S, Mitchell BM, Sullivan DE, Morris CA and Puschett JB: Marinobufagenin inhibits proliferation and migration of cytotrophoblast and $\mathrm{CHO}$ cells. Placenta 29(3): 266-273, 2008.

21 Uddin MN, Horvat D, Glaser SS, Mitchell BM and Puschett JB: Examination of the cellular mechanisms by which marinobufagenin inhibits cytotrophoblast function. J Biol Chem 283(26): 17946-17953, 2008.

22 Ravindran J, Prasad S and Aggarwal BB: Curcumin and cancer cells: how many ways can curry kill tumor cells selectively? AAPS J 11(3): 495-510, 2009.

23 Indran IR, Tufo G, Pervaiz S and Brenner C: Recent advances in apoptosis, mitochondria and drug resistance in cancer cells. Biochimica et Biophysica Acta 1807(6): 735-745, 2011.

24 Afroze SH, Sloan J, Osuji GA, Drever N, Pilkinton K, Zawieja DC, Kuehl TJ and Uddin MN: Cinobufotalin impedes Sw. 71 cytotrophoblast cell line function via cell cycle arrest and apoptotic signaling. Molecular Cell Biochem 422(1-2): 189-196, 2016.

25 Uddin MN, Horvat D, DeMorrow S, Agunanne E and Puschett JB: Marinobufagenin is an upstream modulator of Gadd45a stress signaling in preeclampsia. Biochimica et Biophysica Acta 1812(1): 49-58, 2011.

26 Ehrig JC, Horvat D, Allen SR, Jones RO, Kuehl TJ and Uddin $\mathrm{MN}$ : Cardiotonic steroids induce anti-angiogenic and antiproliferative profiles in first trimester extravillous cytotrophoblast cells. Placenta 35(11): 932-936, 2014.

27 Ehrig JC, Afroze SH, Reyes M, Allen SR, Drever NS, Pilkinton KA, Kuehl TJ and Uddin MN: A p38 mitogen-activated protein kinase inhibitor attenuates cardiotonic steroids-induced apoptotic and stress signaling in a Sw-71 cytotrophoblast cell line. Placenta 36(11): 1276-1282, 2015.

28 Zhao HY, Wu FK, Qiu YK, Wu Z, Jiang YT and Chen JY: Studies on cytotoxic constituents from the skin of the toad Bufo bufo gargarizans. J Asian Nat Prod Res 12(9): 793-800, 2010.

29 Bagrov AY, Shapiro JI and Fedorova OV: Endogenous cardiotonic steroids: physiology, pharmacology, and novel therapeutic targets. Pharmacol Rev 61(1): 9-38, 2009.

30 Tsurimoto T: PCNA binding proteins. Front Biosci 4: 849-858, 1999.

31 Kholmukhamedov A, Schwartz JM and Lemasters JJ: Mitotracker probes and mitochondrial membrane potential. Shock (Augusta, Ga.) 39(6): 543, 2013.
Received June 19, 2018

Revised August 20, 2018

Accepted August 22, 2018 